\title{
败血症休克大鼠心功能及心肌肌浆网 钙转运的变化*
}

\author{
吉勇董林旺 ${ }^{(1)}$ 吴玲 唐朝枢 ${ }^{(1)}$ 苏静怡 $^{(1)}$ \\ (北京医科大学病理生理教研室, (1) 心血管基础研究所. 北京 100083)
}

\section{关键词败血症休克 心功能 肌浆网 钙 受体}

败血症休克多伴有不同程度的心功能障碍, 严重影响休克的转归和预后, 其发生机理至今 尚未阐明. 有研究表明, 休克时心功能障碍与心肌细胞钙稳态失衡有关 ${ }^{[1,2]}$. 心肌肌浆网 (Sarcoplasmic reticulum, SR) 是调节胞浆游离铨浓度的重要细胞器, 它通过快速摄取和释放 $\mathrm{Ca}^{2+}$ 对心肌舒缩活动产生重要影响. 本工作选用大鼠腹膜炎败血症休克模型, 应用蔗糖密度 梯度离心法制备大鼠心肌 SR 膜, 观察休克不同阶段心功能以及 SR 钙摄取和钲释放功能的 变化, 探讨心肌 SR 钲转运功能变化在心功能障碍中的发病学意义.

\section{1 材料与方法}

\section{1 败血症休克模型复制}

雄性 Wistar 大鼠 $250 \sim 300 \mathrm{~g}$, 术前禁食 $12 \mathrm{~h}$, 自由饮水, 按 Chaudry 等 ${ }^{[3]}$ 建立的盲肠结扎穿 孔法 (Cecal ligation and puncture, CLP) 复制大鼠腹膜炎败血症休克模型. 动物随机分 3 组, 每组 8 例: (1) 假手术组, 除不结扎盲肠和穿孔外, 其余操作同休克组; (2) 早期休克组, CLP 术后 $9 \mathrm{~h}$;(3) 晚期休克组, CLP 术后 $18 \mathrm{~h}$. 监测 3 组动物血压和心功能的变化, 并分别取心肌制 备心肌 SR 膜.

\section{2 蔗糖密度梯度离心法制备大鼠心肌 SR 膜}

将 Pessah ${ }^{[4]}$ 和 Feher ${ }^{[3]}$ 等人方法加以改良. 速取心脏, 加 A 液 (mmol/L: 蔗糖 $300, \mathrm{HEPES}$ 10 , DTT 0.5, pH7.2) 制备心室肌匀浆, $10000 \times g$ 离心 $20 \mathrm{~min} \times 2$, 收集 2 次离心之上清液, $138000 \times g$ 离心 $40 \mathrm{~min}$, 悬浮沉淀并缓慢加注于蔗糖梯度最上层界面上 (蔗糖密度分别为 $0.8,1.0,1.2 \mathrm{~mol} / \mathrm{L}$ ) $196000 \times g$ 离心 $90 \mathrm{~min}$ 后分取 $0.8 \sim 1.0 \mathrm{~mol} / \mathrm{L}$ 和 $1.0 \sim 1.2 \mathrm{~mol} / \mathrm{L}$ 交界层悬浮物用 $\mathrm{B}$ 液 $(\mathrm{mol} / \mathrm{L}:$ $\mathrm{KCl} 600$, 咪唑 $10, \mathrm{NaN}_{3}$ 5, DTT 0.5, pH7.2) 稀释后, $138000 \times \mathrm{g}$ 离心 $40 \mathrm{~min}$, 沉淀悬于 A 液待用.

Lowry 氏法测定心肌 SR 膜蛋白含量; Jones 法 (定磷法) 测定 SR $\mathrm{Ca}^{2+}-$ ATP ase 活性.

\section{$1.3 \mathrm{SR}^{45} \mathrm{Ca}^{2+}$ 摄取测定}

${ }^{45} \mathrm{Ca}^{2+}$ 摄取反应体系中含 $0.5 \mathrm{mmol} / \mathrm{L}$ EGTA, 参照游离 $\left[\mathrm{Ca}^{2+}\right]$ 计算软盘提供的数据加人 ${ }^{45} \mathrm{CaCl}_{2}$, 控制反应液中游离 $\left[\mathrm{Ca}^{2+}\right]$ 为 $5 \mu \mathrm{mol} / \mathrm{L} . \mathrm{SR}$ 膜蛋白 $20 \mu \mathrm{g}$ 取 自 $0.8 \sim 1.0 \mathrm{~mol} / \mathrm{L}$ 蔗糖交

1993-12-23 收稿, 1995-01-06 收修改稿

*国家自然科学基金资助项目 
界层. 以加与不加 $3 \mathrm{mmol} / \mathrm{L}$ ATP 反应的滤膜 ${ }^{45} \mathrm{Ca}^{2+}$ 放射活性之差代表 ATP 依赖的 $\mathrm{SR}{ }^{45} \mathrm{Ca}^{2+}$ 摄取量.

\section{$1.4 \mathrm{SR}\left|{ }^{3} \mathrm{H}\right|$ ryanodine 受体结合测定}

反应液中含 $0.5 \mathrm{mmol} / \mathrm{L} \mathrm{EGTA}$, 游离钙浓度为 $50 \mu \mathrm{mol} / \mathrm{L}$, 加人 $2.5 \sim 75 \mathrm{nmol} / \mathrm{L}$ 的 $\left[{ }^{3} \mathrm{H}\right]$ ryanodine (放射比活性 $50000 / \mathrm{min} \cdot \mathrm{pmol}^{-1}$ ), 非标记 ryanodine 浓度 $10 \mu \mathrm{mol} / \mathrm{L}$, 与 $40 \mu \mathrm{g} \mathrm{SR}$ 膜蛋白 (取自 $1.0 \sim 1.2 \mathrm{~mol} / \mathrm{L}$ 蔗糖界面层) $37^{\circ} \mathrm{C}$ 反应 $30 \mathrm{~min}$. 测定滤膜 $\left[{ }^{3} \mathrm{H}\right]$ 的放射活性, 计算 特异性 $\left[{ }^{3} \mathrm{H}\right]$ ryanodine 受体结合量以 $\mathrm{pmol} / \mathrm{mg}$ 蛋白表示.

统计学处理: 各组之间比较用方差分析 $q$ 检验, 结果以均值土标准误表示.

\section{2 实验结果}

\section{1 败血症休克不同阶段心功能的变化}

败血症休克早期 (CLP 术后 $9 \mathrm{~h}$ ), 心率、左心室 $+\mathrm{dp} / \mathrm{dt}_{\text {max }}$ 升高, 晚期时 (CLP 术后 $18 \mathrm{~h}$ ) 降 低; 左心室 $-\mathrm{dp} / \mathrm{dt}_{\text {max }}$ 和平均动脉压早期没有变化, 晚期降低; 左心室舒张末期压力 (LVEDP) 早期有升高趋势, 晚期显著升高. 表明随休克进展 CLP 大鼠心功能逐渐减退, 如表 1 所示.

表 1 败血症休克不同阶段心功能的变化 $\left(\bar{X} \pm S_{\overline{\mathrm{X}}}, n=7\right)$

\begin{tabular}{|c|c|c|c|}
\hline 指 标 & 假手术组 & 早期休克组 & 晚期休克组 \\
\hline 心摔 次 $\cdot \min ^{-1}$ & $396 \pm 8$ & $509 \pm 6^{\mathrm{b})}$ & $242 \pm 23^{b l}$ \\
\hline $\mathrm{LV}+\mathrm{dp} / \mathrm{dt}_{\max } / \mathrm{kPa} \cdot \mathrm{s}^{-1}$ & $687 \pm 15$ & $809 \pm 17^{b 3}$ & $491 \pm 25^{b}$ \\
\hline $\mathrm{LV}-\mathrm{dp} / \mathrm{dt}_{\max } / \mathrm{kPa} \cdot \mathrm{s}^{-1}$ & $645 \pm 18$ & $622 \pm 14$ & $348 \pm 17^{(0)}$ \\
\hline LVEDP $/ \mathrm{kPa}$ & $0.5 \pm 0.1$ & $0.9 \pm 0.2$ & $2.0 \pm 0.3^{3)}$ \\
\hline $\mathrm{MABP} / \mathrm{kPa}$ & $16.0 \pm 0.5$ & $16.1 \pm 0.7$ & $12.6 \pm 0.8^{b)}$ \\
\hline
\end{tabular}

a) $P<0.05$

b) $P<0.01$ 与假手术组比较

\section{2 心肌 $\mathrm{SR} \mathrm{Ca}^{2+}-$ ATPase 活性及 $\mathrm{Ca}^{2+}$ 摄取功能的变化}

败血症休克早期大鼠心肌 SR $\mathrm{Ca}^{2+}-$ ATPase 活性无明显变化, 晚期休克组心肌 SR $\mathrm{Ca}^{2+}-$ ATPase 活性明显降低, 较假手术组降低 40\%( $p<0.01)$, 较早期休克组低 $31 \%(p<0.01)$, 见表 2 .

表 2 败血症休克时心肌 SR 功能的变化 $\left(\bar{X} \pm S_{\overline{\mathrm{X}}}, n=8\right)$

\begin{tabular}{|c|c|c|c|}
\hline \multirow{2}{*}{ 组 别 } & \multirow{2}{*}{$\mathrm{Ca}^{2+}-$ ATPase 活性/ $\mu \mathrm{mol} \mathrm{Pi} \cdot\left(\mathrm{mg} \cdot \mathrm{h}^{-1}\right)^{-1}$} & \multicolumn{2}{|c|}{$\left[{ }^{3} \mathrm{H}\right]$ ryanodine 受体结合 } \\
\hline & & $B_{\max } / \mathrm{pmol} \cdot \mathrm{mg}^{-1}$ & $K_{\alpha} / \mathrm{nmol} \cdot \mathrm{L}^{-1}$ \\
\hline 假手术组 & $40.8 \pm 1.9$ & $6.6 \pm 0.7$ & $12.4 \pm 0.7$ \\
\hline 早期休克组 & $35.5 \pm 2.5$ & $4.1 \pm 0.1^{\mathrm{a})}$ & $7.9 \pm 0.2^{a)}$ \\
\hline 晚期休 克组 & $24.4 \pm 2.4^{3)}$ & $3.9 \pm 0.1^{33}$ & $10.4 \pm 1.0$ \\
\hline
\end{tabular}

a) $p<0.01$ 与假手术组比较

图 1 示心肌 SR 摄 $\mathrm{Ca}^{2+}$ 时间曲线. 假手术组 SR 在第 $1 \mathrm{~min}$ 内摄取 $\mathrm{Ca}^{2+}$ 的量, 即钙摄取 初速率为 $(50 \pm 4) \mathrm{nmol} / \mathrm{mg} \cdot \mathrm{min}^{-1}$, 随反应时间延长, SR 摄 $\mathrm{Ca}^{2+}$ 量增加, $15 \mathrm{~min}$ 时达饱和, 
摄 $\mathrm{Ca}^{2+}$ 量为 $(132 \pm 14) \mathrm{nmol} / \mathrm{mg}$. 早期休克组 SR 铮摄取初速率低于假手术组 $41 \%(p<0.01)$, $5 \mathrm{~min}$ 后 $\mathrm{SR}$ 摄 $\mathrm{Ca}^{2+}$ 量接近假手术组水平。晚 期休克组心肌 SR 钙摄取初速率和最大摄钻量 分别较假手术组降低 $59 \%(p<0.01)$ 和 $28 \%$ $(p<0.01), \mathrm{SR}$ 摄 $\mathrm{Ca}^{2+}$ 量亦低于早期休克组水 平.

\section{3 心肌 $S R\left[{ }^{3} H \mid\right.$ ryanodine 受体结合功能的 变化}

图 2示 $\left[{ }^{3} \mathrm{H}\right]$ ryanodine 与心肌 $S R$ 钻释放通道 ryanodine 受体结合的 Scatchard 作图. 测得假 手术组大鼠心肌 $S R\left[{ }^{3} \mathrm{H}\right]$ ryanodine 受体结合最 大值 $\left(B_{\max }\right)$ 为 $(6.6 \pm 0.7) \mathrm{pmol} / \mathrm{mg}$ 蛋白, $K_{\mathrm{d}}$ 值为 $(12.4 \pm 0.7) \mathrm{nmol} / \mathrm{L}$. 早期休克组心肌 SR $\left[{ }^{3} \mathrm{H}\right]$ ryanodine 受体结合量明显减少, $B_{\text {max }}$ 值较假手术组低 $38 \%(p<0.01), K_{\mathrm{d}}$ 值为 $(7.9 \pm 0.4) \mathrm{nmol} / \mathrm{L}$, 亦低于 假手术组水平 $(p<0.01)$ (见表 2$)$. 晚期休克组 $B_{\text {max }}$ 值较假手术组降低 $41 \%(p<0.01)$, 与早期 休克组没有显著差别, $K_{\mathrm{d}}$ 值较假手术组无明 显变化, 但高于早期休克组水平 $(p<0.05)$ (表 2).

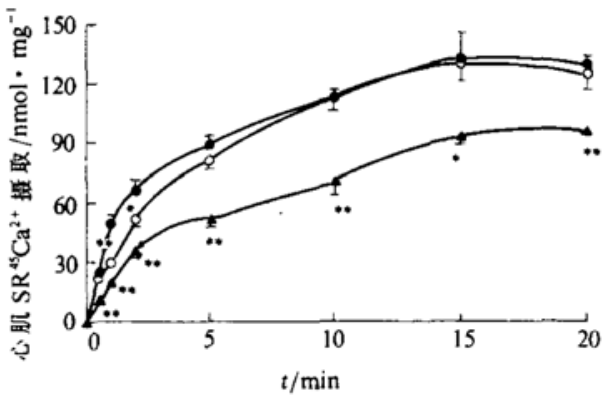

图 1 败血症休克时心肌 SR 摄 ${ }^{45} \mathrm{Ca}^{2+}$ 时间曲线 - 为假手术组, $O$ 为早期休克组, $\Delta$ 为晚期休克组 ${ }^{*} p<0.05,{ }^{* *} p<0.01, n=8$

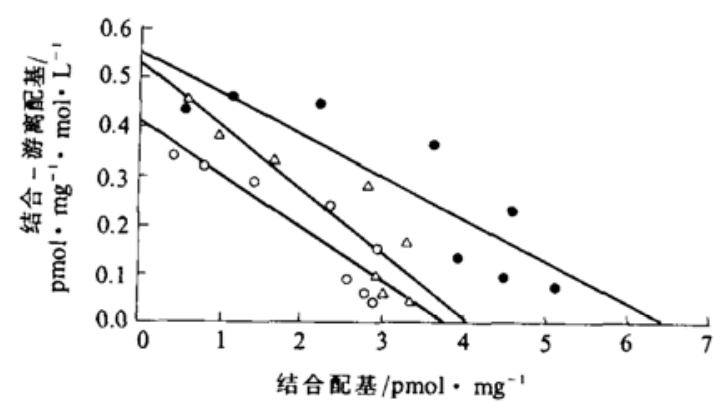

图 2 心肌 SR $\left[{ }^{3} \mathrm{H}\right]$ ryanodine 受体结命Seatchard 作泈 - 为假手术组, $\triangle$ 为早期休克组, $\bigcirc$ 为晚期休克组

\section{3 讨论}

败血症休克时常伴有心功能障碍. 我们观察到 CLP 大鼠早期 (9h) 呈现高动力循环状 态, 晚期 (18h) 处于低动力循环状态; 心脏舒缩功能减弱, 血压下降. LVEDP 在休克早期有升 高趋势, 心室舒张末期压力升高是心功能减退较早出现的变化之一, 结果提示早期休克大鼠 心功能开始减退, 并随休克进展逐渐恶化. 这与一些文献报道的结果相符 $[6.7]$.

近年一些研究表明, 休克时心肌细胞钙稳态失衡与心功能障碍的发生密切相关 ${ }^{[1.2]}$. 心肌 $\mathrm{SR}$ 通过快速摄取和释放 $\mathrm{Ca}^{2+}$ 在维持心肌细胞钲稳态以及心肌舒缩功能上起重要作用. 本工 作发现早期休克大鼠心肌 SR $\mathrm{Ca}^{2+}$ 摄取初速率降低, 晚期休克大鼠除摄 $\mathrm{Ca}^{2+}$ 率减低外, $\mathrm{SR}$ 摄 $\mathrm{Ca}^{2+}$ 量和 $\mathrm{Ca}^{2+}-\mathrm{ATPase}$ 活性均明显降低, 表明随休克进展 $\mathrm{SR} \mathrm{Ca}^{2+}$ 摄取功能损伤加剧. 心肌 SR 摄 $\mathrm{Ca}^{2+}$ 速率降低, 将减慢 $\mathrm{Ca}^{2+}$ 与肌钲蛋白解离的速率, 引起心室肌舒张延缓, 心功 能表现为 $-\mathrm{dp} / \mathrm{dt}_{\max }$ 降低; $\mathrm{SR}$ 摄 $\mathrm{Ca}^{2+}$ 量减少将导致舒张末期胞浆游离 $\left[\mathrm{Ca}^{2+}\right]$ 升高, 促进心肌细胞 䥻超载的发生. Yoshida 等人发现, SR $\mathrm{Ca}^{2+}$-ATPase 活性降低与心肌细胞䥻聚积程度呈显著 正相关 ${ }^{[8]}$. 提示心肌 SR 摄钙功能减低和 $\mathrm{Ca}^{2+}-$ ATPase 活性减弱在休克时心肌细胞钙稳态失衡 乃至心功能障碍发生上具有重要作用.

心肌 $\mathrm{SR} \mathrm{Ca}^{2+}$ 释放经由 $\mathrm{Ca}^{2+}$ 释放通道 - ryanodine 受体完成 ${ }^{[9]}$, 因此测定 SR ryanodine 受体功能在一定程度上反映了 $\mathrm{SR} \mathrm{Ca}^{2+}$ 释放功能. 败血症休克早、晚期, 大鼠心肌 $\mathrm{SR}\left[{ }^{3} \mathrm{H}\right]$ 
ryanodine 受体结合 $B_{\text {max }}$ 值降低, 表明 SR 上可结合的 ryanodine 受体数目减少. 提示由于休 克时 $\mathrm{SR} \mathrm{Ca}^{2+}$ 释放通道减少, 收缩期 $\mathrm{SR}$ 的 $\mathrm{Ca}^{2+}$ 释放量减低,影响心肌兴奋 - 收缩偶联, 导致 心肌收缩力减弱, 心功能表现为 $+\mathrm{dp} / \mathrm{dt}_{\text {max }}$ 降低.

本工作观察到败血症休克过程中心肌 SR $\mathrm{Ca}^{2+}$ 摄取和 $\mathrm{Ca}^{2+}$ 释放功能的变化与整体实验 中大鼠心功能改变基本一致, 提示心肌 SR $\mathrm{Ca}^{2+}$ 转运功能障碍引起的心肌细胞钙稳态失衡是 心功能障碍发生发展的重要因素之一。

\section{参考文献}

I Hulsman W C. Lamers J M, Stam J R H et al. Calcium overload in endotoxemia. Life Sci, 1981. 29: $1009 \sim 1014$

2 Parker J I, Adams H R. Isolated cardiac preparations: model of intrisic myocardial dysfunction in circulatory shock. Circ Shock. 1985, 15: $227 \sim 245$

3 Chaudry 1 H, Wichterman K A, Baue A E. Effect of sepsis on tissue adenine nucleotide levels. Surgery, 1978, 85: $205 \sim 211$

4 Pessah $\mathrm{I}$ N, Duire E L, Schiedt $\mathrm{M}$ J et al. Anthraquinone-sensitized $\mathrm{Ca}^{2+}$ release channel from rat cardiac sarcoplasmic reticulum: possible recptor-mediated mechanism of doxorubicin cardiomyopathy. Mol Pharmacol, 1990. 37: $503 \sim 508$

5 Feher J J. Davis M D. Isolation of rat cardiac sarcoplasmic reticulum with improved $\mathrm{Ca}^{2+}$ uptake and ryanodine binding. J Mol Cell Cardiol, 1991, 23: 249 258

6 Sneil R J. Parrillo J E. Cardiovascular dysfunction in septic shock. Chest, 1991, 99: 1000 1009

7 Sugi K. Cardiac dysfunction after acute endotoxin administration in conscious sheep. Am J Physiol, 1991, 260: HI $474 \sim 1481$

8 Yoshida Y. Degradation of sarcoplasmic reticulum calcium pumping ATPase in ischemia-repurfused myocardium: role of calcium activated neutral protease. Basic Res Cardiol, 1990, 85: 495 507

9 Inui M, Saitio A, Fleischer S. Isolation of the ryanodine receptor from cardiac sarcoplasmic reticulum and identity with the feet structures. J Biol Chem, 1987. 262: $15637 \sim 15642$ 